\title{
Domain walls collision in Fe-rich and Co-rich glass covered microwires
}

\author{
A. Chizhik ${ }^{1}$, J.M. Blanco ${ }^{2}$, M. Ipatov ${ }^{1}$, V. Zhukova ${ }^{1}$, J. Gonzalez ${ }^{1}$, A.Zhukov ${ }^{1,3}$ \\ ${ }^{1}$ Dpto. Fisica de Materiales, Fac. Quimicas, UPV/EHU, 20018 San Sebastian, Spain \\ ${ }^{2}$ Dpto. de Física Aplicada, EUPDS, UPV/EHU, 20018, San Sebastian, Spain \\ ${ }^{3}$ IKERBASQUE, Basque Foundation for Science, 48011 Bilbao, Spain
}

\begin{abstract}
We report the results of the investigation of domain walls propagation in Fe-rich and Co-rich microwires performed using Sixtus-Tonks and magneto-optical Kerr effect techniques. It was found that under certain experimental conditions we are able to create the regime of the motion of two domain walls moving to opposite directions which terminates by the collision of the domain walls. Also the domain walls collision was visualized using magneto-optical Kerr effect microscope when the surface giant Barkhausen jump induced by circular magnetic field has been observed.
\end{abstract}

\section{Introduction}

Domain walls (DW) dynamics of the in magnetic microwires has become a subject of much interest due to their outstanding magnetic properties. Magnetic domain walls are used to store information in small magnetic devices $[1,2]$. Consequently, a deep understanding of the process of the magnetization reversal results to be the key for future applications in these devices.

The domain walls collision has been studied in glass covered Fe-rich mocrowires [3] using the experimental set-up with two synchronized nucleation coils that gives great possibility of the control of domain nucleation.

In this paper we manipulate the DW dynamics in cylindrical microwires by the controlled DWs collision. In order to demonstrate this phenomenon we have chosen Fe-rich and Co-rich microwires having different types of the magnetic structure.

\section{Experimental details}

Experiments have been performed in microwires of the nominal compositions $\mathrm{Fe}_{74} \mathrm{~B}_{13} \mathrm{Si}_{11} \mathrm{C}_{2}$ (Sample 1 metallic nucleus radius $7.3 \mu \mathrm{m})$ and $\mathrm{Co}_{67} \mathrm{Fe}_{3.85} \mathrm{Ni}_{1.45} \mathrm{~B}_{11.5} \mathrm{Si}_{14.5} \mathrm{Mo}_{1.7}$ (Sample 2 - metallic nucleus radius $11.2 \mu \mathrm{m}$ and Sample 3 - metallic nucleus radius $10.7 \mu \mathrm{m})$. The domain structure of Fe-based amorphous microwires consists of single large axial domain with magnetization oriented axially and the external domain structure with radial magnetization at the surface [4]. The magnetization switching is therefore related with the propagation of the single head-to head DW along the wire inside the single-domain inner core. Therefore for studying of the DW dynamics the Sixtus-
Tonks experiments are the most appropriate [6]. Magnetic field is generated by solenoid applying rectangular shaped voltage. We used 3 pick-up coils, mounted along the length of the wire and propagating DW induces electromotive force (emf) in the coils. These emf sharp peaks are picked up at an oscilloscope upon passing the propagating wall.

The domain structure of Co-based amorphous microwires consists of single large axial domain with magnetization oriented axially and the external domain structure with circular or helical magnetization at the surface. During the experiments, in order to produce the circular magnetic field an electric current flowing through the wire has been used.

Magnetic domain imaging has been performed in Corich microwire by means of optical polarizing microscopy in the longitudinal magneto-optic Kerr effect (L-MOKE) configuration [6]. The magnetic contrast of the domain structure was improved by standard image processing. The circular magnetic domains could be observed basically because of different in-plane components of the surface magnetization that transforms to black-white contrast when the polarized light reflects from the cylindrical-shape surface of the microwire. The images of the domain structures obtained by L-MOKE microscopy show the difference of in-plane magnetization components.

\section{Experimental results and discussion}

Distribution of the local nucleation fields along the sample length indicates that the nucleation field near wires ends is considerably smaller (actually there already exist the reversal closure domains which appear to 
minimize the stray field energy). This characteristic feature reflected by the significant difference in nucleation field near the samples ends and far from the sample ends is responsible for the origin of single DW propagation. The re-magnetization runs by de-pinning and consequent fast DW propagation from one of the wire ends.

In the Sixtus-Tonks experiments we observed sharp voltage peaks induced by propagating DWs in the pickup coil surrounding the microwire (Sample 1) (Fig. 1).

Moving the sample with the holder inside the magnetization coil and/or applying the bias field $\mathrm{H}_{\mathrm{b}}$
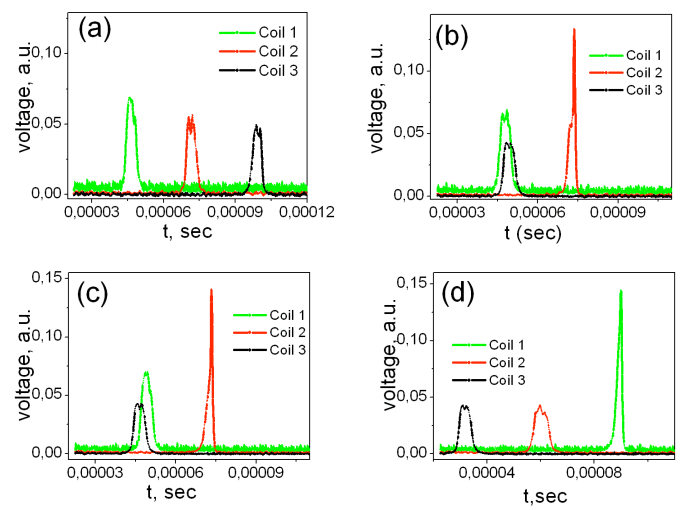

Fig. 1. Voltage peaks induced by the propagating DW in the 3 pick-up coils in Sample 1 microwires measured at $\mathrm{H}=140 \mathrm{~A} / \mathrm{m}$ with different values of bias magnetic field, $\mathrm{H}_{\mathrm{b}}$ : (a) $0 \mathrm{~A} / \mathrm{m}$, (b) $204 \mathrm{~A} / \mathrm{m}$, (c) $252 \mathrm{~A} / \mathrm{m}$, (d) $740 \mathrm{~A} / \mathrm{m}$.

orientated with some angle with the respect to the wire axis, we can activate the DW propagation from the opposite wire end. Examples of manipulation of DW dynamics are presented in Fig. 1, where time dependence of the voltage peaks in coils $1,2,3$ placed along the 10 $\mathrm{cm}$ long samples and separated by $27 \mathrm{~mm}$ are shown. The application of bias magnetic field oriented with the angle about $5^{\circ}$ with respect to wire axis, can activate the propagation of the DW from the opposite wire end.
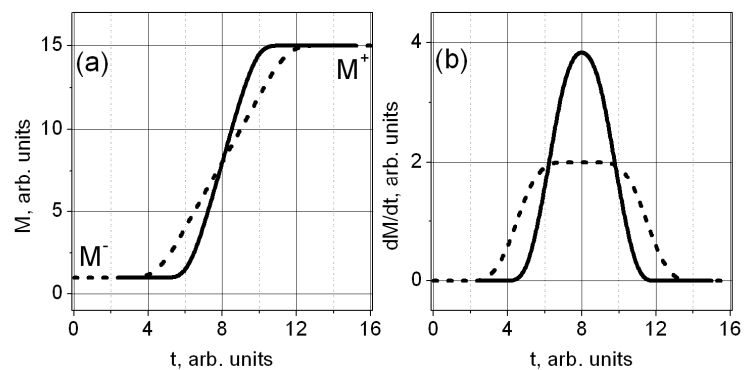

Fig. 2. Schematic pictures related to modelling. (a) Change of magnetization associated with domain walls motion though the coil 2: dashed line - single domain wall motion, solid line two domain walls motion. (b) time derivatives of the mentioned change of magnetization corresponding to the signal in the coil 2: dashed line - single domain wall motion, solid line - two domain walls motion.

As can be appreciated from Fig. 1 (a)-(d), changing value of $H_{b}$ we can activate by controllable way the DW propagation and induce the DWs collision in different places of the sample. If this collision happens under the pick-up coil it will be observed as a voltage peak of increased amplitude. We were able to observe the DWs collision in the position of the 2-nd coil. In this case the height of the signal from coil 2 drastically increases (Fig. 1 (b)-(c)). Additionally, when the $H_{b}$ value is high enough, the DW moving from the opposite sample end arrives to the coil 1 even before the DW starting the propagation from the nearest sample end (Fig. 1(d)). It is with mentioning, that similar effect we observed when we used the magnetizing coil shorter than the sample. In this case when we moved the sample inside the magnetizing coil we were able to activated DW propagation from one side of the sample or from the other one.

To explain two times growth of the voltage peak related to the DWs collision we performed a modelling of the signal in the pick-up coil, at the moment of DWs collision (Fig. 2). The change of the magnetic flux from $\mathrm{M}^{-}$to $\mathrm{M}^{+}$related to the single DW motion is presented as a dashed line in the Fig. 2(a).

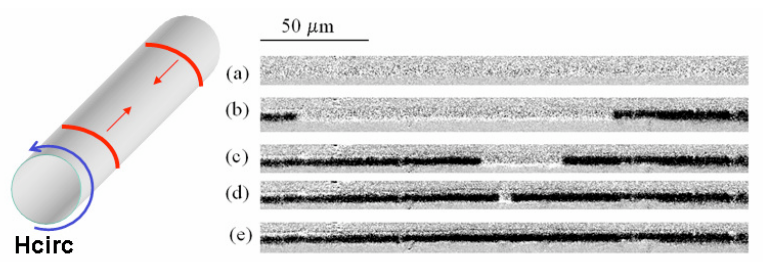

Fig. 3. Images of circular domain walls collision, in the electric current $I=0.38 \mathrm{~mA}$, recorded at different times: (a) $t=0$; (b) $t=62 \mathrm{~ms}$; (c) $t=124 \mathrm{~ms}$; (d) $t=186 \mathrm{~ms}$; and (e) $t=248 \mathrm{~ms}$.

The magnetic flux changes two times faster (solid line in the Fig. 2(a)) when the magnetization reversal occurs in the form of two domains wall motion toward each other instead of single DW motion. The main idea of our modelling is that two DWs move simultaneously in some special moment of the collision through the coil 2 that causes the increase of the voltage peak. Taking into account that the voltage signal in the coil is the time derivative of the change of the magnetic flux $d \Phi / d t$ (Fig. 2(b)) two time increase of the velocity of the change of the magnetic flux in the moment of the DW collision reflects in double growth of the voltage peak (Fig 2(b), solid line). Another words, the higher induced voltage is due to the faster change of the magnetic flux.

The domain collapse has been fixed for the first time in conventional Fe-rich wire [7]. Therefore, our present experiments confirm the common nature of domain walls dynamics in conventional and glass-covered wires.

In the Sample 2 we have visualised using the MOKE microscopy the extensive motion of two circular domain walls in the surface of the microwires. This motion terminates by the collapse of one of the circular domains (Fig. 3). The surface domain structure transformation was obtained after applying of circular magnetic field with a constant value of amplitude and increasing time duration pulses. Additionally, a small nucleation coil was placed at the end of the wire in order control the domain walls propagation along the wire. To realize the collision of two DWs we varied the phase of the magnetic field in the nucleation coil jointly with the electric current flowing along the microwire.

Also the study of the controlled domain nucleation and domain walls motion has been performed in Co-rich microwire (Sample 3) which in the helical magnetic 
structure has been discovered earlier [8]. The experiments have been performed by the Sixtus-Tonks method with 3 secondary coils.

When the applied axial magnetic field was spatially homogeneous three jumps in three secondary coils take place at the same moment of the time (Fig. 4(a)). It means that the magnetization reversal in the microwire occurs as a rotation of the magnetization mainly homogeneously along the microwire. The introduction of weak local inhomogeneity of magnetic field changes the experimental picture: the splitting of the three peaks is observed (Fig. 4(b)). It means that in this experimental configuration, the magnetization reversal occurs as a directed motion of the domain wall. The presence of the spatial magnetic local inhomogeneity causes the local nucleation of domain wall at the end of microwire.
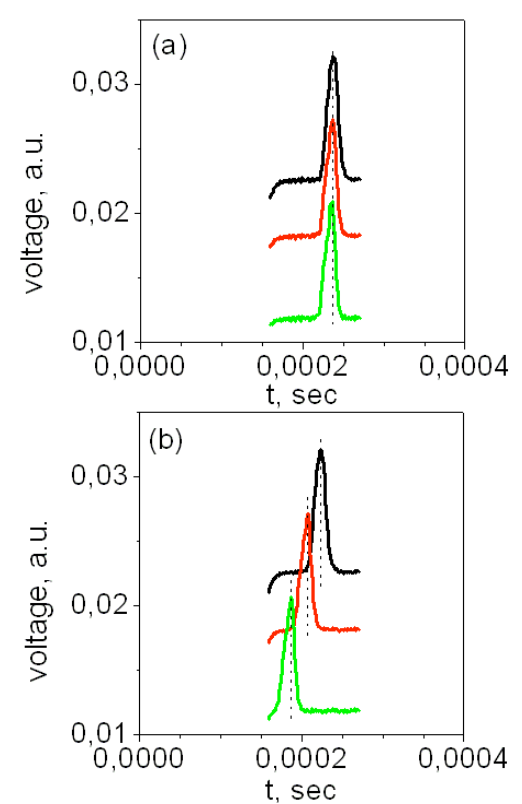

Fig. 4. Results of Sixtus-Tonks experiments. Jumps in three secondary coils: (a) homogeneous axial magnetic field, (b) axial magnetic field with local un-homogeneity.

\section{Conclusion}

We have performed three types of magnetic and magneto-optical experiments to demonstrate the variety of the controllable domain walls motion and collision in glass covered microwires. It aws found that the collision is followed by the collapse of the unfavorable domain. Under certain experimental conditions it is possible to manipulate the domain walls dynamics in a field-driven regime. The collision was fixed as in the volume and in the surface of the microwire. For the first time the visualization of surface DW collision has been realized. The volume collision was recognized as a sharp growth of the electric signal in the secondary coil. This growth has found clear explication in the frame of the model presuming the simultaneous motion of two domains along the secondary coil. The manipulation with the microwire containing the helical magnetic structure, permitted to demonstrate that the presence of small magnetic inhomogeneity could cardinally transform the magnetic behaviour - the homogeneous magnetization reversal is replaced by the unidirectional motion of the solitary domain wall.

\section{References}

1. G. S. D. Beach, C. Nistor, C. Knutson, M. Tsoi, J. L. Erskine, Nature Mater., 4, 741 (2005)

2. D. Atkinson, D. S. Eastwood, L. K. Bogart, Appl. Phys. Lett., 92, 022510 (2008)

3. T.-A. Óvári, M.Tibu, H. Chiriac, IEEE Trans. Magn., 47, 2838 (2011)

4. A. Zhukov, Appl. Phys. Let., 78, 3106 (2001)

5. V. Zhukova, J. M. Blanco, M. Ipatov, A. Zhukov, IEEE Trans. Magn., 47, 3783 (2011)

6. A. Chizhik A. Zhukov, J. Gonzalez, J. M. Blanco, Phys. Status Solidi A, 2082277 (2011)

7. L.V. Panina, M. Mizutani, K. Mohri, F.B. Humphrey, I. Ogasawara, IEEE Trans. Magn., 27, 5331 (1991)

8. A. Chizhik, C. Garcia, A. Zhukov, J. Gonzalez, P. Gawronski, K. Kulakowski, J.M. Blanco, Appl. Phys. Lett., 103, 07E742 (2008) 